\begin{tabular}{|c|c|c|}
\hline Case Reports in & \multicolumn{2}{|c|}{ Case Rep Gastroenterol 2016;10:199-206 } \\
\hline astroenterology & $\begin{array}{l}\text { DOI: } 10.1159 / 000445976 \\
\text { Publisnea onme: 1VIray 19, } 2016\end{array}$ & $\begin{array}{l}\text { (c) } 2016 \text { The Author(s) } \\
\text { Published by S. Karger AG, Basel } \\
\text { www.karger.com/crg }\end{array}$ \\
\hline
\end{tabular}

\title{
Ten-Year Survival of a Patient Treated with Stereotactic Gamma Knife Radiosurgery for Brain Metastases from Colon Cancer with Ovarian and Lymph Node Metastases: A Case Report
}

\author{
Nobuhiro Morinaga ${ }^{a} \quad$ Naritaka Tanaka $^{a}$ Yoshinori Shitara ${ }^{a}$

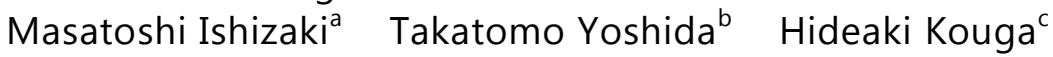 \\ Kazuki Wakabayashic ${ }^{c}$ Minoru Fukuchi $^{d}$ Yoshiyuki Tsunoda $^{\mathrm{e}}$ \\ Hiroyuki Kuwano \\ ${ }^{a}$ Department of Surgery, Fujioka General Hospital, Fujioka, Japan; ${ }^{b}$ Department of \\ Pathology, Fujioka General Hospital, Fujioka, Japan; 'Department of Neurosurgery, Fujioka \\ General Hospital, Fujioka, Japan; ${ }^{d}$ Department of Digestive Tract and General Surgery, \\ Saitama Medical Center, Saitama Medical University, Moroyama, Japan; \\ ${ }^{\mathrm{e}}$ Tsunoda Hospital, Tamamura, Japan; ${ }^{\mathrm{f}}$ Department of General Surgical Science, \\ Graduate School of Medicine, Gunma University, Maebashi, Japan
}

\section{Keywords}

Colorectal cancer - Brain metastasis - Stereotactic Gamma Knife radiosurgery . Multidisciplinary therapy

\begin{abstract}
Brain metastasis from colorectal cancer is infrequent and carries a poor prognosis. Herein, we present a patient alive 10 years after the identification of a first brain metastasis from sigmoid colon cancer. A 39-year-old woman underwent sigmoidectomy for sigmoid colon cancer during an emergency operation for pelvic peritonitis. The pathological finding was moderately differentiated adenocarcinoma. Eleven months after the sigmoidectomy, a metastatic lesion was identified in the left ovary. Despite local radiotherapy followed by chemotherapy,
\end{abstract}


Morinaga et al.: Ten-Year Survival of a Patient Treated with Stereotactic Gamma Knife Radiosurgery for Brain Metastases from Colon Cancer with Ovarian and Lymph Node Metastases

the left ovarian lesion grew, so resection of the uterus and bilateral ovaries was performed. Adjuvant chemotherapy with tegafur-uracil (UFT)/calcium folinate (leucovorin, LV) was initiated. Seven months after resection of the ovarian lesion, brain metastases appeared in the bilateral frontal lobes and were treated with stereotactic Gamma Knife radiosurgery. Cervical and mediastinal lymph node metastases were also diagnosed, and irradiation of these lesions was performed. After radiotherapy, 10 courses of oxaliplatin and infused fluorouracil plus leucovorin (FOLFOX) were administered. During FOLFOX administration, recurrent left frontal lobe brain metastasis was diagnosed and treated with stereotactic Gamma Knife radiosurgery. In this case, the brain metastases were well treated with stereotactic Gamma Knife radiosurgery, and the systemic disease arising from sigmoid colon cancer has been kept under control with chemotherapies, surgical resection, and radiotherapy.

(C) 2016 The Author(s)

Published by S. Karger AG, Basel

\section{Introduction}

In general, patients with brain metastasis from colorectal cancer show a poor prognosis, with an overall median survival of 2.8-5.4 months [1-4]. Several reports have mentioned that neurosurgical resection has a favorable impact on prognosis. In previous reports from Japan, only 5 patients with solitary brain metastasis survived more than 5 years [5, 6]. Of these 5 patients, 4 had undergone surgery with chemo- and/or radiotherapy. Whereas surgical resection seemed to be a favorable prognostic factor, it was not statistically significant [7]. So control of systemic disease from colorectal cancer is also important for long-term survival in patients with brain metastasis.

In this report, we present a case of multiple brain metastases, lymph node metastasis, and ovarian metastasis from sigmoid colon cancer treated with chemotherapies, surgical resection, and radiotherapy. The first brain metastatic lesions were treated with stereotactic Gamma Knife radiosurgery (GKS). Because of the less invasive nature of GKS, recurrent brain metastatic lesions could repeatedly be treated with GKS and systemic chemotherapy could be administered. At the time of writing, in March 2016, the patient is alive, with neither neurological nor systemic symptoms. The multidisciplinary therapy, including GKS, resulted in the patient's survival for 10 years after the identification of the first brain metastases and survival for 13 years and 7 months after the sigmoidectomy.

\section{Case Report}

A 39-year-old woman underwent an emergency drainage operation for pelvic peritonitis in August 2002. The left ovary was swollen, and paraovarian inflammation was apparent. A stenotic lesion existed at the sigmoid colon, and intraoperative colonoscopy revealed the stenosis to be due to sigmoid colon cancer. Sigmoidectomy with D1 lymph node dissection and stoma construction was therefore performed on the sigmoid colon. The pathological finding was moderately differentiated adenocarcinoma (fig. 1a), and the UICC TNM classification was T2, Nx, M0. Adjuvant chemotherapy was provided using carmofur.

In July 2003, left ovarian metastasis and para-aortic lymph node metastasis were diagnosed and treated using fluorouracil and folinic acid (leucovorin) (5-FU/LV). After 4 months, the para-aortic lymph node metastasis was considered to have shown complete response, but the ovarian metastasis was classified as stable disease. Although chemotherapy was 
changed to irinotecan for 5 months and tegafur/gimeracil/oteracil potassium (TS-1) for 12 months, the ovarian metastasis grew. We applied radiotherapy to the left ovarian lesion. All these therapies proved ineffective in controlling the ovarian metastasis, so resection of the uterus and bilateral ovaries was performed in July 2005. The pathological finding was adenocarcinoma, similar to the sigmoid colon cancer (fig. 1b). Both the sigmoid colon cancer and the left ovarian tumor were stained immunohistochemically with cytokeratin (CK) 7 and CK20. The sigmoid colon cancer showed negative staining for CK7 and positive staining for CK20, as is usually seen in the majority of colorectal cancers (fig. 1c, e). The left ovarian tumor also showed negative staining for CK7 and positive staining for CK20, compatible with its colon cancer origin (fig. 1d, f).

Adjuvant chemotherapy with tegafur-uracil (UFT)/calcium folinate (leucovorin, LV) was initiated. In November 2005, the right cervical subcutaneous lymph nodes became palpable. Computed tomography revealed swelling of the right cervical and mediastinal lymph nodes, so these lesions were diagnosed as lymph node metastases. In March 2006, convulsions of the left arm and leg were noted, followed by weakness of the left leg as well as gait disturbance. Magnetic resonance imaging (MRI) showed metastases in the bilateral frontal lobes (fig. 2a). We selected GKS for the treatment of brain metastases. After GKS, the patient was able to walk and the tumors were reduced in size (fig. 2b). In April 2006, radiotherapy for cervical and mediastinal lymph nodes was performed. In June 2006, administration of oxaliplatin and infused fluorouracil plus leucovorin (FOLFOX) was started as adjuvant chemotherapy. During the administration of FOLFOX, recurrence of a brain metastasis in the left frontal lobe was noted (fig. 3a). In November 2006, GKS for the recurrent brain metastasis was performed and was effective in controlling the lesion (fig. 3b). After 10 courses of FOLFOX, chemotherapy was not administered. At the time of writing, in March 2016, 10 years after the identification of the first brain metastasis, the patient has neither neurological nor systemic symptoms.

\section{Discussion}

Brain metastasis is infrequent in colorectal cancer. Patients with brain metastasis from colorectal cancer show a poor prognosis, with an overall median survival of 2.8-5.4 months [1-4]. As stated in case reports from Japan of colorectal cancer patients with solitary brain metastasis, only 5 patients survived more than 5 years [5, 6]. Reports of long-term survival with multiple brain metastases from colorectal cancer are not found in the literature.

In a retrospective review of patients treated for brain metastases from colorectal cancer, Suzuki et al. [4] reported that absence of extracranial metastatic lesions, 1 or 2 brain metastatic lesions, and treatment with neurosurgical resection represent favorable prognostic factors. In addition, several reports have mentioned that patients treated with surgery survived longer than those who did not undergo surgery [1-3]. Whereas resection of brain metastasis seemed to be a favorable prognostic factor, it was not identified as statistically significant in the multivariate analysis by Bartelt et al. [7]. In a study by Mongan et al. [8], the survival curve according to treatments suggests longer survival with sterotactic radiosurgery group than resection plus external beam radiation therapy, WBXRT (whole-brain external-beam radiation therapy), or WBXRT plus chemotherapy group. All of these reports involved retrospective studies, so selection biases may not have been excluded. In the analysis of 12 cases of brain metastasis from colorectal cancer reported by Tajima et al. [9], 9 patients showed multiple lesions. The longest survivor was a patient who had multiple lesions 
Morinaga et al.: Ten-Year Survival of a Patient Treated with Stereotactic Gamma Knife Radiosurgery for Brain Metastases from Colon Cancer with Ovarian and Lymph Node Metastases

and survived 505 days (median overall survival: 107 days). This patient was treated with chemotherapy and radiotherapy without surgery.

In patients with a solitary brain metastasis from colorectal cancer who underwent surgery with or without postoperative radiotherapy, death occurred in $82 \%$ of cases, according to the evolution of the systemic disease [10]. Thus, control of the systemic disease is important for long-term survival in patients with brain metastasis. Complete resection of brain metastases does not seem necessary for the long-term survival of patients with brain metastasis from colorectal cancer.

Petrovich et al. [11] demonstrated the efficacy and safety of GKS in selected patients with brain metastasis from melanoma, lung cancer, breast cancer, renal cell carcinoma, colon cancer, and others. In their study, depending on the intracranial tumor volume, disease control rates of $80-90 \%$ were obtained. Rades et al. [12] compared whole-brain radiotherapy (WBRT) plus stereotactic radiosurgery (SRS) to surgical resection (OP) plus WBRT for 1-3 brain metastases from breast cancer, lung cancer, and other tumors. They concluded that WBRT plus SRS appeared to be at least as effective as OP plus WBRT in terms of treatment outcomes.

In the present case, the primary sigmoid colon cancer was resected. The ovarian metastasis was treated with chemotherapy and radiotherapy and finally resected. The lymph node metastases were controlled with radiotherapy and chemotherapy. The brain metastases were well treated twice with GKS. This multidisciplinary therapy enabled the long-term survival of this patient. The clinical course of this case suggests the effectiveness of GKS for treatment of brain metastasis from colon cancer.

Recently, the median survival of patients with metastatic colorectal cancer has been prolonged by the sequential use of combination regimens. Controlling brain metastasis with GKS and using the combination regimens for systemic disease from colorectal cancer can be effective treatment options for certain patients with brain metastasis from colorectal cancer.

\section{Statement of Ethics}

All procedures followed were in accordance with the Helsinki Declaration of 1964 and later revision. Informed consent was obtained from the present patient for being included in this study.

\section{Disclosure Statement}

The authors declare that they have no conflict of interest.

\section{References}

1 Alden TD, Gianino JW, Saclarides TJ: Brain metastases from colorectal cancer. Dis Colon Rectum 1996;39:541-545.

2 Kye BH, Kim HJ, Kang WK, Cho HM, Hong YK, Oh ST: Brain metastases from colorectal cancer: the role of surgical resection in selected patients. Colorectal Dis 2012;14:e378-e385.

-3 Hammoud MA, McCutcheon IE, Elsouki R, Schoppa D, Patt YZ: Colorectal carcinoma and brain metastasis: distribution, treatment, and survival. Ann Surg Oncol 1996;3:453-463.

4 Suzuki Y, Yamaguchi T, Matsumoto H, Nakano D, Honda G, Shinoura N, Karasawa K, Takahashi K: Prognostic factors and treatment effects in patients with curatively resected brain metastasis from colorectal cancer. Dis Colon Rectum 2014;57:56-63. 
Morinaga et al.: Ten-Year Survival of a Patient Treated with Stereotactic Gamma Knife Radiosurgery for Brain Metastases from Colon Cancer with Ovarian and Lymph Node Metastases

5 Yamamoto M, Yoshimitsu M, Mukaida H, Hirabayashi N, Takiyama W, Kaneko M: A long-term survived case of solitary brain metastasis from sigmoid colon carcinoma. J Jpn Surg Assoc 2013;74:1436-1442.

-6 Nishimura M, Akamoto S, Yamamoto N, Fujiwara M, Okano K, Suzuki Y: A long-term surviving case of transverse colon cancer presented with solitary brain metastasis. J Jpn Surg Assoc 2014;75:1355-1359.

-7 Bartelt S, Momm F, Weissenberger C, Lutterbach J: Patients with brain metastases from gastrointestinal tract cancer treated with whole brain radiation therapy: prognostic factors and survival. World J Gastroenterol 2004;10:3345-3348.

-8 Mongan JP, Fadul CE, Cole BF, Zaki BI, Suriawinata AA, Ripple GH, Tosteson TD, Pipas JM: Brain metastases from colorectal cancer: risk factors, incidence, and the possible role of chemokines. Clin Colorectal Cancer 2009;8:100-105.

-9 Tajima Y, Ishibashi K, Ishiguro T, Osawa T, Sakimoto T, Okada N, Miyazaki T, Yokoyama M, Yamano T, Nishimura K, Hondo M, Takahashi T, Ishida H: Analysis of 12 cases of brain metastasis from colorectal cancer (in Japanese). Gan To Kagaku Ryoho 2009;36:2245-2247.

10 D'Andrea G, Isidori A, Caroli E, Orlando ER, Salvati M: Single cerebral metastasis from colorectal adenocarcinoma. Neurosurg Rev 2004;27:55-57.

11 Petrovich Z, Yu C, Giannotta SL, O’Day S, Apuzzo ML: Survival and pattern of failure in brain metastasis treated with stereotactic gamma knife radiosurgery. J Neurosurg 2002;97(suppl):499-506.

12 Rades D, Kueter JD, Veninga T, Gliemroth J, Schild SE: Whole brain radiotherapy plus stereotactic radiosurgery (WBRT+SRS) versus surgery plus whole brain radiotherapy (OP+WBRT) for 1-3 brain metastases: results of a matched pair analysis. Eur J Cancer 2008;45:400-404. 


\section{Case Reports in Gastroenterology}
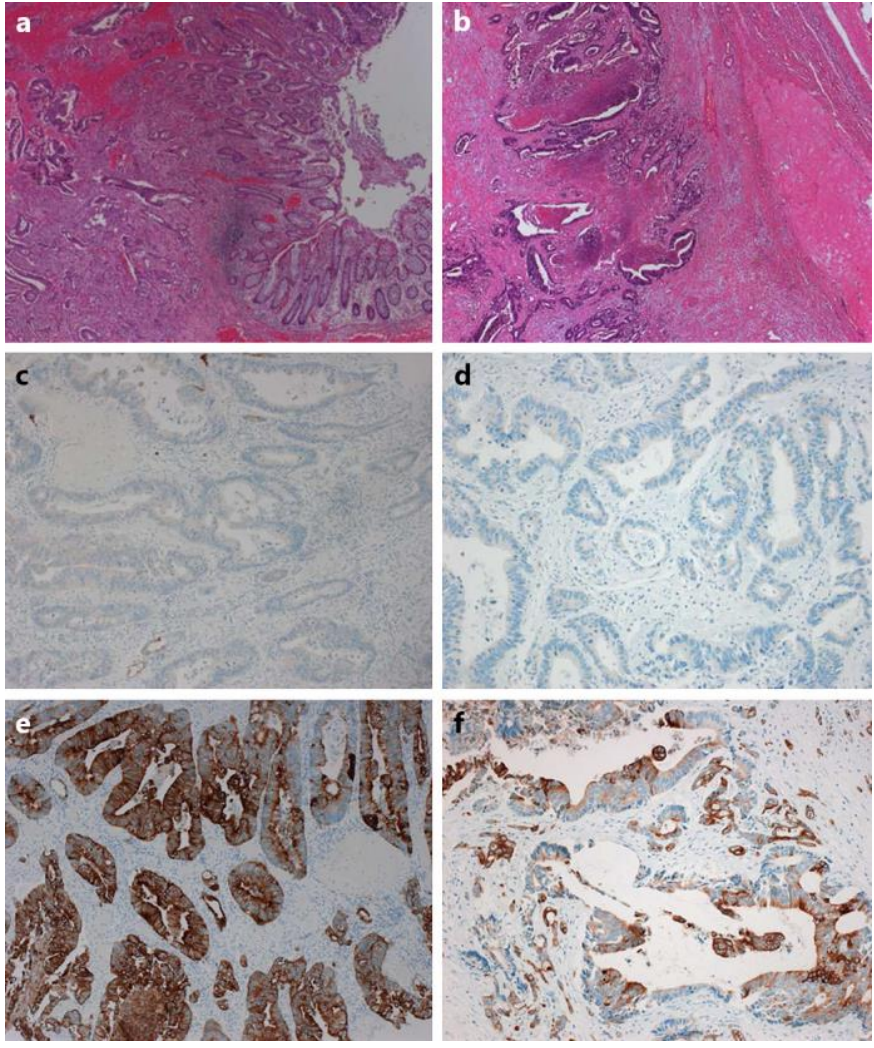

Fig. 1. Pathological findings of primary sigmoid colon cancer and metastatic ovarian tumor. The primary sigmoid colon cancer (a) represents a moderately differentiated adenocarcinoma, and the metastatic ovarian tumor (b) is also identified as an adenocarcinoma. Both the sigmoid colon cancer and ovarian tumor show negative staining for CK7 (c: sigmoid colon cancer; d: ovarian tumor) and positive results for CK20 (e: sigmoid colon cancer; f: ovarian tumor). 


\section{Case Reports in Gastroenterology}
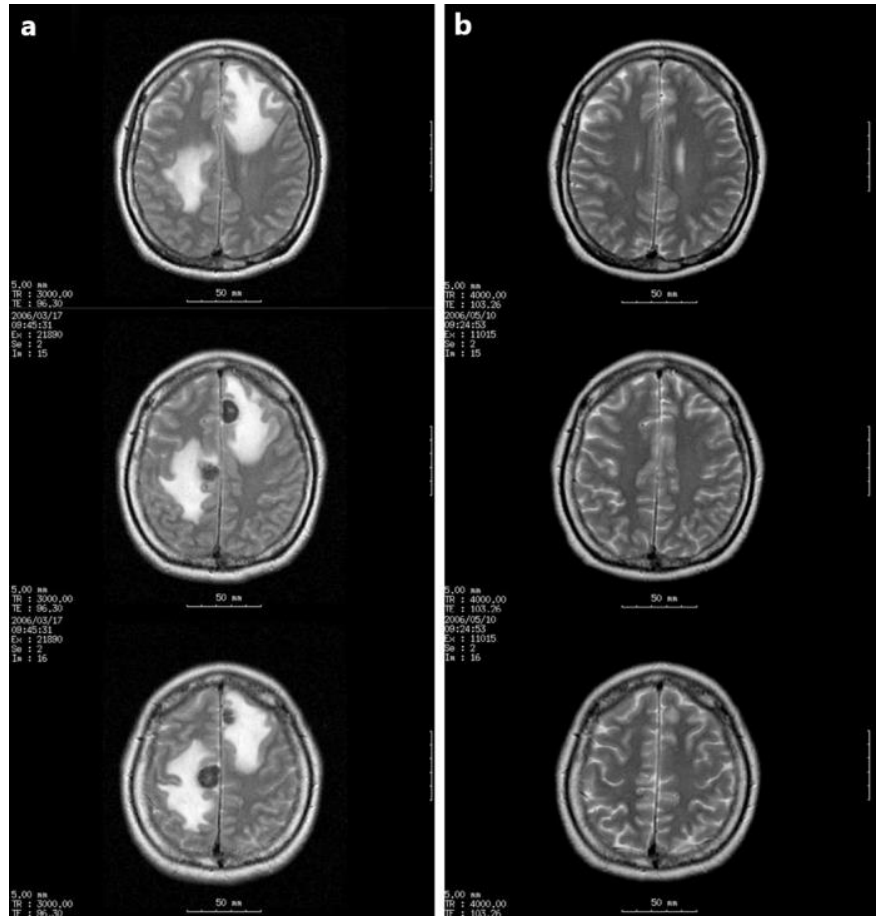

Fig. 2. MRI in March 2006 showed multiple metastatic tumors approximately $18 \mathrm{~mm}$ in diameter as well as surrounding brain edema in the bilateral frontal lobes (a). After GKS, the tumors had reduced in size by May 2006 (b). 


\section{Case Reports in Gastroenterology}
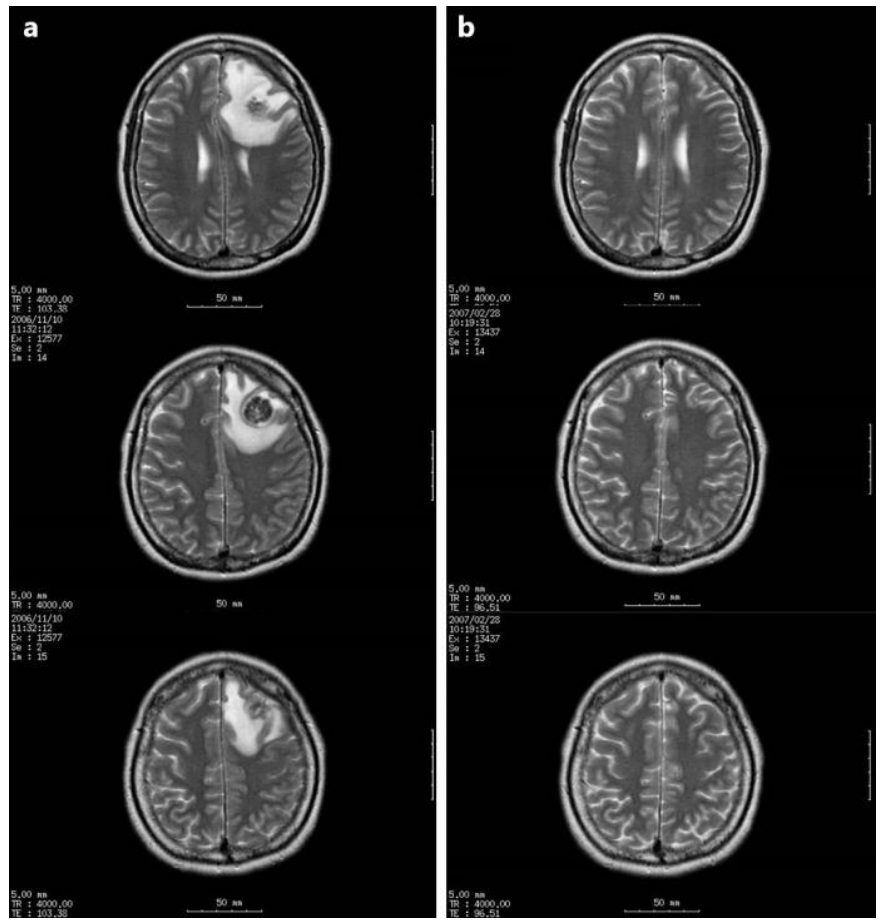

Fig. 3. MRI in November 2006 showed recurrent brain metastasis approximately $20 \mathrm{~mm}$ in diameter in the left frontal lobe (a). MRI in February 2007 showed that GKS was effective in controlling the lesion (b). 